Portland State University

PDXScholar

Winter 2021

\title{
Genome-Wide Sequencing in Clinical Genetic Counseling
}

Sydney Schulz

Portland State University

Follow this and additional works at: https://pdxscholar.library.pdx.edu/honorstheses

Part of the Health Information Technology Commons Let us know how access to this document benefits you.

\section{Recommended Citation}

Schulz, Sydney, "Genome-Wide Sequencing in Clinical Genetic Counseling" (2021). University Honors Theses. Paper 1136.

https://doi.org/10.15760/honors.1167

This Thesis is brought to you for free and open access. It has been accepted for inclusion in University Honors Theses by an authorized administrator of PDXScholar. Please contact us if we can make this document more accessible: pdxscholar@pdx.edu. 


\title{
Genome-Wide Sequencing In Clinical Genetic Counseling
}

\author{
Brianna Burroughs \\ Portland State University Honors College \\ Thesis Advisor Kim H. Brown of the Portland State University Department of Biology
}

\begin{abstract}
Genome-wide Sequencing provides an exciting opportunity for the future of genetic counseling. However, the efficacy of GWS in covering G-C rich regions of DNA, detection of variants, coverage of the exome, reanalysis, and identifying disease origin in non-coding regions of DNA remains to be proven. GWS provides a diagnostic success rate of $36-73 \%$ in pediatric cases and $50-67 \%$ of adult rare disease and alters the path of necessary care offered by genetic counselors. Limitations significantly hamper the success of GWS in a genetic counseling setting, such as difficulties in obtaining consent for testing and data storage, the high rate of incidental findings, cost of testing and insurance coverage, and underrepresentation in high-need populations. Additional large-scale studies are required to provide empirical evidence for the diagnostic ability of GWS along with the development of tools for genetic counselors to effectively provide clinical and psychosocial care for clients and institutional standards for variant calling and the handling of incidental findings.
\end{abstract}

\section{INTRODUCTION}

Since the Human Genome Project's initial publication in 2003, human genome sequencing has evolved far beyond its origins as a multi-year, multi-billion dollar venture. Today genome-wide sequencing (GWS) can be completed for less than a thousand dollars $^{1}$ and yields results in as little as 24 hours $^{2,3}$. Indeed, the availability of genomic sequencing is reflected in the publications of such large-scale studies such as the 10,000 Genome Project, The Cancer Genome Atlas Program, and the UK 100,000 Genomes Project. The American College of Medical Genetics (ACMG) has stated their support of genetic testing and genomic medicine's use in the clinical setting ${ }^{3}$. Genomic analysis has tremendous implications for medicine and has been of particular relevance to the field of genetic counseling. In the last decade, the use of GWS using traditional technologies has been studied for its ability to compete with other next-generation sequencing techniques, ability to identify molecular mechanisms for rare and undiagnosed disorders, identifying suspected genetic illness in neonates, and pharmacogenetic possibilities. Many of these studies take place in a clinical setting where genetic counselors are, or should be, present to ascertain patient consent, inform decisions, and provide psychosocial care prior to and after results have been received. As the availability of genomic sequencing and the public and medical use of such tests increase, genetic counselors will inevitably face genomic medicine in clinical practice. The goal of this review is to identify the state of GWS as a diagnostic tool genetic counselors may use and elucidate the current state of GWS in clinical practice, outlining benefits, best practices, and concerns.

\section{GENOME-WIDE SEQUENCING}

There is a significant gap in the research created by the lack of any large-scale study $(>1000)$ comparing genome-wide sequencing directly against current methodologies, such as targeted next generation sequencing (NGS), chromosomal microarray (CMA), or whole-exome sequencing (WES). In the limited current literature, most studies compare only the rate at which GWS provides a diagnosis of pathogenic variants within the exome, rather than the overall diagnostic ability or inclusion of portions of the genome not covered by WES. While it is critical to affirm the diagnostic comparability of GWS, neglecting any diagnosis from within non-coding regions of DNA is to ignore the clear possible advantage of this method. Despite the gap in research, this section will discuss the current state of GWS and analyze the claims made by proponents of GWS that it is better than current standards at analyzing G-C rich regions of DNA, variant detection (CVNs and SNVs), covers exon regions more completely, is superior in identifying the cause of disease, and has a significant advantage over other methods due to the ability to identify mutations in non-coding regions of the genome and the availability of re-sequencing.

Guanine-Cytosine Rich Regions

One of the most notable improvements to genome-wide sequencing responsible for increasing clinical utility was the development of polymerase chain reaction (PCR)-free techniques. Although the use of PCR is known to result in amplification bias due to the process of recombination ${ }^{2,4}$, it is the primary method for assembling data in WES, and only one study evaluating the 
efficacy of PCR-free WES was found by the author ${ }^{5}$. Using PCR for GWS has been shown to be less effective than WES at correctly mapping G-C rich sections of the exome ${ }^{6,7}$. WES only targets roughly $1-2 \%$ of the genome, creating a much smaller margin of error when compared to GWS which covers $>90 \%$ of the genome ${ }^{8}$. This PCR-free method of sequencing is the strongest case made for the improvement of $\mathrm{G}-\mathrm{C}$ rich region capture by GWS. A 2015 study comparing 48 WES samples against 52 GWS samples from different individuals found GWS performed slightly better across G-C rich regions except in where content was exceptionally high: in this case GWS capture also suffered. This study was limited in cohort size as well as sequencing reliability, due to using data from multiple sources. The authors note that the lower rate of WES was possibly tied to the number of PCR steps used in the sequencing technique which varied across samples ${ }^{9}$. A 2017 study analyzing areas of reduced coverage in 400 patients who underwent WES and 100 who received GWS showed areas of high G-C content to reduce coverage but did not specify the difference in efficacy between the two sequencing techniques ${ }^{10}$. A larger study from the same year by Carss et al. (2017) studied 722 sets of patient data. The majority (650) of these patients had GWS in addition to or instead of WES, and showed GWS to be significantly more effective at covering the genes with a high G-C content (45-65\%) when compared to WES. GWS was able to reach a higher read depth and showed much less variability than WES. Specifically this was used to elucidate a pathogenic variant of $G U C Y 2 D$ which resulted in inherited retinal disease (IRD) that would have been missed by WES alone ${ }^{11}$. The claim that GWS is significantly better at covering $\mathrm{G}-\mathrm{C}$ rich regions than WES has a mechanical basis and shows promise, but more empirical evidence is needed before this claim can be validated.

\section{Superior Variant Detection}

An appealing potential for the use of GWS is an improved rate of detection for single nucleotide variants (SNVs) and structural variants, including copy-number variants (CNVs), indels, and inversions. More sensitive testing has the potential to identify the cause of unknown or undiagnosed genetic diseases ${ }^{12}$ and lead to more effective medical intervention.

In a study of 100 patients, GWS was shown to have a $34 \%$ rate of identifying the cause of genetic disease, a four-fold increase over CMA which achieved diagnosis in only $8 \%$ of patients $^{22}$. A perhaps more convincing meta-analysis from 2018 summarizing 37 studies (total 20,068 patients) found both WES and GWS to be significantly more effective at diagnosing children with suspected genetic disease. The study acknowledges, however, no significant difference in efficacy between WES and GWS in the studies analyzed. It is of note that two of the 37 studies showed a non-significant difference, likely due to the smaller sample size: these studies had a combined cohort of 138 while the average sample size for the other studies was $>500{ }^{17}$.

Truly, the closest competitor to GWS is WES, likely owing to the fact that they both cover the portion of the genome responsible for the majority of disease-causing mutations 13,14 . The diagnostic success rate for WES ranges from $23 \%$ to $87 \%$ and is difficult to generalize as the results range widely depending on size of the study, patient phenotype, presence of consanguinity, and other factors. There is a similar range for GWS patients, also greatly affected by the scope and size of study. Despite these challenges, there are those who claim GWS will soon be the clinical standard as a first-tier diagnostic test for patients with anticipated genetic disorder ${ }^{15,16,17,18}$. Studies have borne this out with varying success. In 2015 both WES and GWS were run on 6 unrelated individuals with congenital asplenia and found that while both tests resulted in a similar number of SNVs, 91\% of those detected by WES were thought to be false positives due to errors in alignment or mapping. While GWS had a lower expected false positive rate, it was still undesirably high at $25 \%$. Despite the small size of the study and the lack of diversity in patients, it suggests GWS may be more effective at accurately identifying SNVs within the exome ${ }^{19}$. In a study by Lionel et al. (2018), analyzing the genomes of 103 individuals with unknown suspected genetic disease GWS was able to diagnose pathogenic variants in $41 \%$ of participants, nine of which were structural variants missed by previous $\mathrm{WES}^{16}$. While the cohort size was still small, the diagnostic rate across seven distinct phenotypes and three inheritance patterns. A 2016 study of patients with IRD compared the diagnostic effect of targeted next-generation sequencing against GWS, finding GWS was able to identify the pathogenic variant in 24 of the 33 patients, more than targeted NGS methods ${ }^{13}$. The identified pathogenic variants included those found in non-coding regions, insertions, and deletions ${ }^{20}$. Another study conducted genome-wide high-resolution SNP array in 99 fetuses displaying a congenital heart defect. A significant number of CNVs were identified in 19 of the fetuses with variants of unknown significance in only $3 \%{ }^{21}$. Additionally, genome-wide association studies (GWAS) show promise in detecting new variants related to illness or disease risk, including 44 areas of interest for major depression ${ }^{23}$, and 170 in breast cancer, which include some non-coding regions ${ }^{24}$.

Not all studies have shown positive indications for GWS, however. The aforementioned meta-analysis ${ }^{17}$ showed no significant difference between WES and GWS. A retrospective analysis by Gross et al. (2019) on 17 individuals who had both CMA and GWS, evaluating the ability of each method to identify CNVs, showed GWS did not perform significantly better than $\mathrm{CMA}^{18}$. A retrospective analysis out of Saudi Arabia of patients with unknown genetic disease showed the limited utility of GWS, as there was not a significant benefit over using WES. GWS was able to identify seven pathogenic variants out of 108 , whereas WES identified five ${ }^{25}$. In a study of 100 individuals, GWS outperformed WES in some respects but was not significantly better at identifying pathogenic variants in the exome ${ }^{9}$. A review by Wilson, Miller, and Rousseau (2017) stated the analytical validity of GWS is weak, due to instability of technical platforms and lack of quality regulation across platforms as well as the range of interpretation methodologies ${ }^{26}$. A 2017 study provided an array of possibilities for text functionality to create a unifying significance of variants, but so far no one system has been employed enough to achieve unity across clinics ${ }^{27}$. 


\section{More Complete Exon Coverage}

The use of PCR in sequencing is relevant to exome analysis as well as in the analysis of G-C rich regions of DNA. In addition to improving the areas of high GC content, using PCR-free GWS methods allows for greater general coverage of the exon region of the genome $e^{25,28}$. A 2014 study showed GWS was able to cover $74.8 \%$ of the exome at a depth of $40 x$, much higher than the $48 \%$ 40x depth accomplished by WES ${ }^{29}$. However, some disagree with this claim and report exome coverage at $100 \mathrm{x}$ for $\sim 95 \%$ for WES and GWS 30x for the entire genome ${ }^{30}$. This is likely due to discrepancies in technology at the time of publication. Other studies point to the uniformity of coverage made possible by GWS to make their claim that GWS will replace WES, stating that the methods required to create similar uniformity in WES would be complex ${ }^{6}$. GWS is also said to be free of bias when compared to WES, and a 2015 study analyzed both methods with three different metrics. WES showed bias in all three metrics, while GWS did not show bias 9 . However, one study claimed to find no significant relationship between the coverage of sequencing and ability to diagnose pathogenic variants ${ }^{31}$. Yet another study showed GWS to do "considerably better" than WES at sequencing the exome with the ability to achieve a $95 \%$ rate of SNV detection with a uniform average of 14 reads $^{2}$. Some of the discrepancies in the data may result from varying use in technology and from a conflicting method of interpreting variants. In 2015, the ACMG updated their guidelines on variant calling standards which may clear up some confusion in subsequent studies $^{32}$. However, the wide range of results indicates a significant need for clear guidelines on sequence calling as well as additional large-scale studies assessing PCR-free WES coverage against GWS coverage across the entire exome.

\section{Ability to Reanalyze Data for New Diagnosis}

Proponents of GWS in a clinical setting have mentioned the potential benefit of resequencing the genomes of patients who did not receive a diagnosis in the first round of testing. Storing genomic data and periodically reinvestigating it for variants discovered in the intervening years without having to reorder testing or inconvenience a patient, additionally reducing cost, time, and discomfort of conducting targeted gene panels and other tests to target variants of formerly unknown significance, has obvious appeal ${ }^{15,}{ }^{16}$. Reassessment of existing data is no more difficult than the original analysis ${ }^{33}$. Many patients without diagnosis are identified to have a variant of unknown significance (VUS). These may be reclassified over time with more information to be benign, likely benign, likely pathogenic, or pathogenic. The knowledge base around genomic information is rapidly accumulating, suggesting that new publications and the classification of VUS could lead to new diagnosis and identification of molecular pathogenicity if genomic patient data is regularly re-evaluated.

In a study of GWS in 100 children with an undiagnosed disorder, only $36 \%$ received a diagnosis on the first test. Despite ongoing clinical care, new diagnoses were made only when the genomic data was reanalyzed two years later, yielding seven more diagnoses. From this research, the authors suggest this 5 -fold increase over CMA and 3-fold increase in diagnostic yield compared to other sequencing methods warrants reanalysis of genomic data for pediatric patients with undiagnosed genetic disease every 1-2 years until a diagnosis is found or the phenotype progresses ${ }^{33}$.

Although reanalysis of GWS has the added advantage over CMA, targeted gene panels, and WES due to the volume of data: When the whole genome is present there is the ability to identify genes which had not been targeted by panels or WES, such as the $H M G A 2$ gene $^{33}$. However, this is a slight advantage as new genes are rarely identified. Some evidence suggests reanalysis of WES is no more effective at diagnosing unidentified disease than GWS. For example, the previously discussed study out of Saudi Arabia from Alfares et al. (2018) also measured the effect of reanalysis. In their study, reanalyzing all 108 genomes resulted in the diagnostic yield for GWS decreasing from nine to seven, four of which were also identified by reanalyzing the WES data $^{25}$. Considering the discrepancy in the difficulty of reanalyzing an exome versus a genome, the authors suggest it is more efficient to reanalyze any available exome data before running or reanalyzing GWS. One study reanalyzed existing genomic and exomic data for 371 patients with developmental delay (DD) after three years. The original success rate in diagnosis for GWS was higher (26.2\%) than WES (22\%), but after the interval of three years, WES showed a significantly higher identification rate of $11 \%$, while GWS only identified $1.6 \%{ }^{34}$. With these findings, the authors propose a 6-part framework for reanalysis which includes strategies for reanalysis, prioritization, timelines, and data-sharing techniques.

It is not clear if GWS reanalysis has significant advantage over reanalysis of WES. A 2019 study conducted reanalysis of data sets from two studies. In the first, manual reanalysis of 250 patients' exome data yielded a diagnostic rate of $46.8 \%$. Despite this high rate of success, the authors caution that manual reanalysis may not be feasible in large-scale projects, as this was conducted over a period of five years by a team of staff. In the second study, 2000 patient exome data was analyzed through an automated process and is possibly responsible for the smaller diagnostic yield of $36.7 \%{ }^{35}$. In another study, 101 exomes of patients who had died of unexplained sudden death or idiopathic disease were reanalyzed after 1-7 years, identifying 3 new variants for the rare disease patients and 1 sudden death case $^{36}$.

\section{Mutation Identification in Non-Coding Regions}

Another enticing aspect of GWS is the potential for illuminating diseases caused by mutations in non-coding regions of DNA ${ }^{9,15}$. The ENCODE project determined a large portion of the non-coding genome is active ${ }^{37}$, and mutations in regulatory elements such as promoters, enhancers, operons, silencers, non-coding RNA, or insulators could lead to disease $\mathrm{e}^{38,39,40}$. Mutations in non-coding regions are also associated with tumorigenesis, such as the TERT telomerase promoter region which is present in $\sim 70 \%$ of melanomas ${ }^{41}$. This presents a clear advantage over WES and would be most competitive with targeted NGS. However, the non-coding regions of the human 
genome and the mechanisms of action are still being researched ${ }^{38}$. This method would rely heavily on large, population-specific reference panels in order to be effective, the existence of which is limited but growing ${ }^{40}$. This method of analysis presents hope for individuals with unknown cancers and suspected genetic disease, but is not yet a viable clinical option. The development of large-scale population-specific data banks, as well as thorough analysis across a diverse range of populations to identify the biological mechanisms of non-coding DNA, will need to be established before the impact of GWS on diagnosis through the use of noncoding regions can be understood. Without this required background information, promising the results of elucidating molecular causes for disease through analysis of non-coding regions of DNA would be making a false promise.

\section{USE IN CLINICAL PRACTICE}

In this section the current state of genome-wide sequencing in clinical practice will be evaluated, specifically assessing the current efficacy of GWS in pediatric care and rare disease diagnosis as well as what the evidence says on the role of the genetic counselor in genomic interventions.

\section{Use in Pediatric Medicine}

Much of the research conducted on the efficacy of genome-wide sequencing is within the pediatric population. Neonates and young children with genetic illness may not express all clinical features of the disorder which limits facilitation of diagnosis through current hypothesis-guided practices, such as targeted $\mathrm{NGS}^{22,42}$. Additionally, the rapid pace of disease progression establishes a demand for diagnosis sooner rather than later $^{43}$. Having a confirmed diagnosis serves to alter medical intervention, either guiding more effective treatments or halting unnecessary ones ${ }^{3}$. The wide array of information gained by GWS combined with the lowering cost and short processing time of such tests creates an opportunity for hope in a uniquely stressed population.

In the 2018 study mentioned previously (see: Superior Variant Detection), a cohort of 103 pediatric patients with undiagnosed genetic disease were tested to compare GWS against other traditional testing methods. To qualify for the study patients must have undergone single-gene testing, CMA, WES, or another NGS test without reaching diagnosis or likely pathogenic variants. GWS was able to identify the origin of disease in $41 \%$ of the cohort. Additionally, 70 participants also underwent WES for comparison which was outperformed by $\mathrm{GWS}^{16}$. The authors also suggest due to rapidly accumulating information regarding VUS those in the cohort who did not receive diagnosis should have their genomic data reanalyzed every 2-3 years. The same year, a study from a dysmorphology clinic of 60 pediatric patients showed a diagnostic success rate of $68.3 \%$ and included SNVs, and CNVs. The description of the manner in which these diagnosis resulted in alteration of the patient's clinical treatment was particularly illuminating. It included referrals to specialists, imaging, voiding muscle biopsy, and in once case of a family who received a diagnosis of neuronal ceroid lipofuscinosis, a transfer to palliative care ${ }^{44}$. Farnaes et al. (2018) found a diagnosis in $43 \%$ of 42 critically ill infants using GWS, and the genomic data was used additionally to avoid morbidity in $26 \%$ of all infants, major morbidity in $10 \%$, and reduced the likelihood of mortality in $2 \%$, reducing cost for both the providing hospital and families. Additionally, GWS was correlated with reducing time spent in hospital by an estimated 124 days as well as time spent in intensive care units. The presence of GWS altered treatment modalities for 13 of the children, which included starting new medications, stopping medications for an anticipated diagnosis that was wrong, and altering three major surgery plans ${ }^{45}$. This demonstrates the effect not only of correct diagnosis through GWS, but that the elimination of certain disease possibilities can alter care and reduce morbidity in a vulnerable population. A similarly small cohort study of 35 critically ill infants showed a diagnosis of genetic illness through GWS in $57 \%$ of patients, with four patients who achieved diagnosis receiving positive effects on medical management. An additional six patients received a diagnosis that influenced palliative care decisions. Among the diagnosed newborns the 120-day mortality rate was 57\%, a number difficult to quantify as no other studies identified included this data ${ }^{46}$. It should be noted, however, that older studies such as Stravropoulos et al. (2015) did not indicate a significantly higher success rate of GWS compared to WES in a study of 100 pediatric patients ${ }^{22}$. Finally, a systematic review of next generation sequencing (including WES, GWS, CMA, and Standard testing) on 4698 patients across 14 studies showed a diagnosis rate of $30 \%-73 \%$ while alternative testing methods ranged from $3 \%-40 \%$, and listed their opinion that GWS should be used as a first-tier diagnostic test especially in high-risk newborns who have need for rapid, effective, widespread testing to prevent further morbidity or mortality ${ }^{3}$. While the numbers range greatly, much of the hope is placed within the portion of the population GWS can serve. The ability to reduce morbidity, mortality, alter medical interventions, and inform decision making for a vulnerable population such as critically ill neonates forms the basis of many defenses of GWS use in this capacity ${ }^{47}$. Indeed this reflects the experience of the genetic counselor more closely: while large-scale studies and number-crunching are required to inform the evidence based medicine genetic counselors practice, ultimately the clinical genetic counselor will be working one on one with families, providing individualized medical recommendations and psychosocial support. In this situation the emphasis is placed on serving the patient and their family, even with varying rates of diagnostic success.

The diagnostic possibilities of GWS within this target population raises the question of universal use or inclusion in newborn screening, particularly due to the rapid progression of disease and the subsequently shortened timeline for diagnosis ${ }^{17}$. Using GWS for infants with genetic conditions has benefits beyond a straightforward diagnosis, as this data could be used for pharmacogenetic (choosing treatment plans based on personalized genomic medicine made available through testing) and donation purposes (HLA typing, etc.). In a study of 98 pediatric patients who underwent GWS as part of the diagnostic odyssey a targeted analysis of SNPs and indels around the GYP2D6 gene and other genes also responsible for metabolizing drugs commonly used in 
a clinical setting, providing information that led to clinical changes to treatment in 95 of participants ${ }^{48}$. GWS has capabilities for HLA-typing as well with possibility for deeper immunological analysis as well as more effective tissue and organ donation matching ${ }^{49}$. Some argue NGS should be universally available for all who may benefit ${ }^{42}$, while others caution against immediate launch, instead suggesting more research on which diagnosis actively benefits patients and improving turnaround time for genomic testing and interpretation ${ }^{45}$. Friedman et al. (2017) argues GWS must conclusively prove to be both cost effectiveness and reliably distinguish pathogenic variants from benign, as well as rectify ethical concerns of consent, incidental findings, and safe storage of data (see Limitations) before GWS should be used in newborn screening ${ }^{50}$.

Despite the ACMG recommendation against using GWS for fetal testing, the field is evolving. These tests are primarily conducted through amniocentesis or chorionic villus sampling and can be used as both a diagnostic tool and mechanism of precision medicine, despite the logistical challenges and ethical dilemmas associated with such testing ${ }^{51,52,53}$.

\section{Use in Rare Disease Diagnosis}

The second-most popular area of research into the efficacy of GWS is rare disease diagnosis. Sharing much of the appeal with pediatric use, GWS has the potential to end the long and expensive process often referred to as the diagnostic odyssey. If GWS were to show empirical evidence of greater efficacy than WES it would be a boon to this target population. Seventy-nine people comprising 16 distinct families with an undiagnosed genetic illness underwent GWS with a diagnostic success rate of $62.5 \%$. Two of the remaining families received a suspected diagnosis, and four remained unknown. Of the diagnosed diseases, the molecular cause of pathogenicity included missense mutations, nonsense mutations, CNV, SSR, compound heterozygous mutations and a frameshift deletion, across six inheritance patterns. This rate of success was achieved through what they described as steps beyond primary filtering methods, having only detected the cause of mutation in five families. Retrospective analysis and case-by-case study increased the diagnostic yield to $62.5 \%$. The authors note the diagnosis resulted not only in alterations to medical treatment which included targeted medical intervention for children, halting disease progression in some cases, and ended or avoided unnecessary or harmful treatments, but also allowed for prenatal counseling and for action to be taken in a disease which caused early menopause ${ }^{54}$. A study from 2014 conducted GWS on 217 families with an undiagnosed suspected genetic disorder from an extensive range of phenotypes found pathogenic variants in only $21 \%$ of cases. Despite the low rate of detection, the variants included nonsense, missense, noncoding, frameshift, and splicing variants $^{31}$. IRD has been the target of two studies. The first surveyed a phenotypically heterogeneous cohort of 605 patients who had not found the molecular mechanism of disease through other testing, primarily WES or targeted gene panels. GWS identified the pathogenic variant in $56 \%$ of the participants, including variants in $\mathrm{CG}$ rich regions of DNA, deletions, duplications, and three SNVs in noncoding regions of the genome. Nineteen additional genes previously unknown to associate with IRD were identified as possibly contributing to pathogenicity ${ }^{11}$. Due to the complex nature of phenotype and genotype heterogeneity of $\mathrm{RD}$, patient outcomes were not identified as part of this study. However, the inheritance pattern is difficult to ascertain from phenotype alone without confirming the genetic source of illness. It can be reasoned that the knowledge of inheritance patterns could inform patient family planning decisions and decrease morbidity in future generations. The second, smaller study conducted GWS on 52 patients (46 with diagnosed IRD, six control) with a success rate of $50 \%$, and similarly found two additional likely pathogenic variants and three pathogenic variants not detected by targeted NGS. These variants also ranged across protein coding regions, noncoding regions, indels, and new genes of interest ${ }^{20}$. These IRD studies highlight the potential for GWS to improve diagnostic accuracy similar to the claims made throughout the first section of this paper.

\section{Role of the Genetic Counselor}

Despite the claim some studies make that in the near future all health professionals will be faced with genomic technologies and be asked to convey the results and management of genomic testing $^{54,56,57,58}$, it does little to negate the necessity of genetic counselors. The utility of genetic counseling, particularly for pediatric and rare disease populations, is well documented. Patients with children in the neonatal intensive care unit experience higher rates of depression, anxiety, and emotional distress as well as lower coping and perceived social support compared to the general population, These families are in need of the psychosocial counseling genetic counselors are trained to provide $^{56,59}$. Additionally, the acute care setting of the NICU generates differences in patient need from traditional clinical care $^{60}$. The uncertainty and distress faced in this community is similarly found in populations undergoing extensive diagnostic odyssey $^{61}$. Several studies indicate patients who receive genetic counseling pre and post NGS results demonstrate more complete understanding of results, decision making, and less distress than patients who did not receive counseling ${ }^{15,62}$, however others still describe difficulty in "managing expectations and anticipating family response" 61 . One study aimed to develop an effective method for facilitating patient's choices around genome wide sequencing of children with undiagnosed suspected genetic condition. This online tool, DECIDE, combines original and established content from other research projects to balance necessary information with "decision aids" to be used in tandem with genetic counseling and shows a possible direction for effective counseling around GWS ${ }^{64}$. Indeed, the 2014 guidelines from the ACMG states any panel surveying the 56 genes with known pathogenic association require additionally thorough preand post-test counseling for the patient ${ }^{61,65}$.

When a diagnosis is achieved through GWS/NGS, genetic counselors continue to provide psychosocial support navigating the new diagnosis, including empowering medical decision making, social or familial implications, and connecting 
patients with specialists or other local programs ${ }^{56,61,62}$. Other studies highlight the utility of genetic counselors in circumstances where a diagnosis is not reached. Genetic counselors are necessary to provide insight so patients can make informed decisions. Additionally, the emotional distress which can arise at a lack of definitive diagnosis cannot be overlooked. The studies referenced in this paper demonstrate the high risk of one such possibility and can result in feelings of "chaos" and "emotional and financial desperation." To put patients at risk of such outcomes without providing psychosocial support violates ethical nonmaleficence ${ }^{62,66}$.

The increased risk of secondary findings and other consequences of genomic analysis provide some additional difficulties for the genetic counselor ${ }^{67}$ (See: Limitations). One such challenge, although perhaps unexpected, was the development and use of direct to consumer genomic testing. While direct to consumer testing (DTC) often presents an exciting opportunity for patients, they are a poor substitute for GWS offered by licensed clinicians due an absence of regulation in DTC laboratories, issues regarding disclosure of efficacy, and interpretation $^{68}$. This DTC has created an emerging patient population with specific questions clinicians may face. One study conducted by Schmidt et al. discussed the issue of DTC genomic testing and the unique pressure this testing can put on those private practice genetic counselors patients are inevitably faced with. A first hand account from three such genetic counselors, this study examined the experience of dealing with patients who have sought out counseling as a result of tests and interpretations purchased independent of a clinical setting with variable interaction with medical professionals. The average experience of DTC testing is difficult to characterize, as they vary in cost, quality of testing, contact by medical professional, and the manner of information they receive with some tests including interpreted data and others returning raw data which may then be uploaded to other interpretive software, which may or may not be associated with the original company from which the test was purchased. Interpretation from both testing companies and outside crowd sourced reference software vary in regards to the method of identifying and classifying variants. Problems with this system include the lack of uniformity or clinical validity in the interpretive software which has gone through no peer reviewed analysis and provides no explanation for how to interpret the risk of any variation detected. As such, this step is what leads patients into the office of a clinician. In the experience of the counselors interviewed, patients come to a genetic counselor after DTC genomic testing for one of three reasons: they are in good health but seek counseling to be proactive, they have a specific health concern related to family history (such as cancer, heart disease, pathogenic variants, etc.), or they are currently ill and undiagnosed. Each population presents different needs in the genetic counseling setting ${ }^{69}$. A small study from a population focus group suggests patients value the ability to access DTC testing and regulations and should be an area of training for future genetic counselors ${ }^{58}$.

\section{LIMITATIONS}

GWS is not a part of the average genetic counselor's repertoire, and as such there are few genetic counselors trained in gaining consent for and interpreting results of GWS. The cost, turnaround time, storage and informatics burdens, high risk of incidental findings, and the challenge of data interpretation are all areas of concern and hindrance for genetic counselors $22,31,56,70$. Of particular concern is the variance of unknown significance. VUS can be the source of much anxiety for patients, as the specificity of GWS makes it difficult to identify a normal SNV from a pathogenic $\mathrm{SNV}^{62}$, and their status may change from likely benign to likely pathogenic, or from likley pathogenic to benign, as studies further develop ${ }^{30}$. The challenge presented by VUS has been a cause for genetic counselors to avoid work with GWS ${ }^{17,56 \text {, }}$ 62. Additionally, the access to the tools needed for GWS and interpretation are a hindrance for genetic counselors as they are not widely available ${ }^{62}$. Additionally of note is the 2014 recommendation from the ACMG against GWS/WES for prenatal counseling, citing the time of testing as a limitation. While this is a problem for cancer genetic counselors as well, some still do this work $^{71}$

Despite the evident utility of genetic counseling in genome-wide sequencing, there remain logistical, economic, and ethical concerns which must be addressed before GWS can be widely adopted. These include the disparity in establishing patient consent and safe storage of genomic data, handling of incidental findings, expense to the patient, unequal racial representation in reference panels, and direct to consumer testing.

\section{Consent and Data Storage}

Establishing patient consent with large scale testing provides a different challenge than targeted NGS or gene panel testing due to the wider range of data provided, rate of VUS, and implications this information may have for both patient and family members. Consent may be difficult to ascertain due to the complex nature of genomic medicine which often requires more background knowledge than a single gene test or gene panel. A study by Samuel et al. (2017) assessed the efficacy of healthcare professional's ability to inform patients who had genetic or genomic testing between 2013 and 2017 through interviews with 33 patients and a series of focus group discussions with health care professionals (including genetic counselors). The results showed variation in information retention among the patients, some reporting feeling empowered by the knowledge and others only recalling signing a consent form. Commonly patients reported understanding content at the time of appointment but failed to recall important information, such as the consent to sharing relevant data within the family. Patients who were in emotional distress at the time of consultation stated more difficulty in recalling materials discussed, and healthcare providers in the focus group discussed offering a gap between two consultations to allow time for emotional processing. This may be a helpful intervention for patients when the case is not acute. However, it is critical for genetic counselors to be aware of the varying rate of success pre-testing consultation can have and react accordingly. This study closes with a suggested ethical framework 
from their findings, adding to the research on ethical treatment of patients $^{72}$. Studies exploring the complexity of consent and attempting solutions such as narrowing the focus of pre-test consent forms are limited, but present in the literature ${ }^{73,74}$. Current guidelines state the pretest consultation should include the implications of test results for both patient and families, the range of test results possible (benign, likely benign, unknown, like pathogenic, pathogenic), how these results would alter care and future disease risk, as well as discussion of storage of sample and data $^{56}$. These recommendations are an evolving process with both patient and provider input under consideration ${ }^{72,73,74}$. Still, some genetic counselors find this limitation too great and believe it to be a preventative measure for GWS in a genetic counseling clinical setting ${ }^{71}$.

Furthermore, safety concerns regarding storage of genomic data have been expressed by both researchers and patients $^{71,72}$. Genomic information stored for both patients and research participants represents a unique level of risk for patient identification not present in other types of stored data. In addition to the concern of a potential hack, there is a high amount of raw data to store that some have listed may be problematic ${ }^{22,71}$. Considering the rise of cyber attacks targeting hospitals in the last three years alone, the security of genomic data is a pressing concern which must be addressed in those studies that harness $\mathrm{GWS}^{75}$. From this information, pertinent questions are raised. Would the additional security required to protect genomic data come at an increased cost which would limit research/medical availability? How important is privacy over patient care? With a diagnostic rate around $50 \%$, however, this poses a risk to a large portion of the patient population who would receive GWS without an attached diagnosis and be put at increased risk of personal data leaking. The onus is on researchers to produce more data on diagnostic success and morbidity rates in patients with negative GWS diagnosis before this can be ethically deployed.

\section{Incidental Findings}

In addition to ascertaining patient understanding and consent, there is the matter of incidental findings (IFs). Also called secondary findings, these encompass any pathogenic or likely pathogenic results found outside the original reason for testing which create a conflict of medical ethics in the genetic counselor. There are now 67 genes associated with 24 different disorders outlined by the ACMG for monogenic disease, such as Marfan's syndrome and the BRCA1 and BRCA2 genes ${ }^{76}$. Due to the high number of diseases which may be uncovered as IFs, IFs represent a huge range of uncertainty for patients and their families. The risk can range from low to high, disease potential may be mild to severe, and the presentation of illness may occur within several years or towards end of life, if they are to present at all. The task of communicating the potential risks of IFs in order to obtain consent or declination to receive any IFs during GWS provides an additional layer of complexity which must be accounted for when providing genetic counseling. One study, which examined the factors which influence patient attitudes regarding decision-making support from clinicians, identified several crucial elements for clinicians aware of when providing counseling for parents of children who qualify for GWS. These included informational needs, specifically around the process of receiving information rather than content. The majority of participants reported appreciating receiving information in smaller pieces over a longer period of time rather than a "flood" of information. Participants provided suggestions for enhancing support strategies for future patients in their position and were engaged with the collaborative process and described the importance of a trusting and collaborative relationship between patient and provider ${ }^{77}$. IFs have been discussed widely as a complication with WES as well, and similar methodologies for the handling of IFs in WES may be deployed in GWS. IFs are an area of concern especially during the testing of newborns and young children, due to what some call a right to not be burdened with knowledge of potential disease. Diseases such as Alzheimer's or Parkinson's disease which manifests later in life (if at all) can be a cause of medical discrimination due to increased disease risk $^{56,71,78}$. In 2013 the ACMG updated their policy on incidental findings after the National Society for Genetic Counselors and other organizations expressed concern over the original policy stating any IFs found during WES or GWS in the (at the time) 56 genes with associated pathogenicity should be included in all testing reports, irrespective of patient age or reported preference for IFs ${ }^{76}$ in order to reduce morbidity through nonmaleficence. Ultimately the recommendation was updated to allow patients the opportunity to opt out of receiving IFs discovered during WES or GWS. Although they are not official guidelines for practice, the ACMG recommendations are respected and may act as a "temperature check" of the state of the field $^{79}$. Patients may not want to be informed of IFs for a variety of reasons, such as insurance discrimination, cultural stigma, anxiety, or informational overwhelm ${ }^{80}$. Additionally, one study showed parents of children in the NICU who qualified for GWS were significantly less likely to opt to receive IFs when compared to the parents of older children who also qualified for GWS, suggesting there are different motivations in across settings genetic counselors will need to be sensitive to. As the GWS improves diagnostic yield and scope of diagnostic range, the rate of IFs will only increase. A small study evaluating the effect of the ACMG update to recommendations on IFs reported the change did not alter the clinical practice for 26 of the 34 genetic counselors with access to WES interviewed for this study. This was in part due to the preferences in IF reporting in the different laboratories the counselors used. The majority of the laboratories offered counselors the option to opt out of IFs depending on patient preference for such information. The differences cited by interviewed counselors in patient interaction before and after the ACMG recommendations mentioned that the time spent with patients pre-testing increased and the focus of their discussion shifted at least in part. Despite what may sound like a challenge, only $23.5 \%$ of participants considered their sessions post ACMG recommendations to be more difficult than previously. Some cited the sessions became easier now that they had a specific list of IFs to discuss with the patient which they were able to structure the consultation around ${ }^{80}$. This information could be useful for designing institutional protocols for the education of navigating 
discussion around IFs with patients in genomic testing. Additionally, one study highlighted the differences between IFs identified in a clinical setting against those in a research setting. In the research setting, the person identifying the IFs may not be a genetic counselor, physician, or another clinically trained individual to interpret the long-term consequences of IFs or provide the psychosocial counseling appropriate in the context ${ }^{81}$. While institutions will vary in their handling of IFs, there should be recommended protocol genetic counselors can reference in regard to which diseases have significant rates of successful medical interventions/preventions such as those established with BRCA1/BRCA2 and which do not, such as Alzheimers. This might best be accomplished through a databank organizing possible IFs and the status of treatment options, however, as the data and treatment evolve rapidly this would need to be a closely monitored and regularly updated source.

\section{Expense and Insurance}

The cost of GWS continues to decline, with more recent studies acquiring testing for less than a thousand USD per genome ${ }^{54}$. Other studies, however, are quick to establish that this low cost does not take into account the cost of running bioinformatics, the cost of interpretation by a trained clinician, or pretesting and posttesting counseling for patients, which increases the cost by a variable rate depending on the location and resources for the study $^{16}$. Still, the cost-effectiveness of GWS remains difficult to ascertain, as in some cases it may save patients money long-term, as in one pediatric study it was identified the cost of diagnostic testing prior to the study was estimated to be over $\$ 19,000 \mathrm{USD}^{3}$. Given the varying success rate of GWS to produce reliable diagnosis, it is unclear if this would indeed be a cost-effective first-tier diagnostic test for children with unknown genetic disease.

Currently, this is an unattainable option for many patients living in the United States due to the cost of diagnosis and evaluation without the aid of insurance. Although the cost to the patient can be reduced through insurance companies, as of 2021 the largest insurance companies in the United States (by membership): United Healthcare, Anthem, Aetna, Cigna, and Humana $^{82}$. None cover the cost of whole genome sequencing, citing the validity as "undetermined" and "experimental" $83,84,85$, 86,87. United Healthcare specifically states " [GWS] is not Medically Necessary for evaluating any genetic disorder due to the availability of clinically equivalent diagnostic tests." 83 . Despite this, several of these companies are participating in experimental trials which involve the use of GWS. WES is available through most of these plans, but GWS clearly does not have the clinical validity in the eyes of insurance companies to warrant inclusion, even for neonatal cases or those of rare undiagnosed diseases. This provides a significant challenge to the use of GWS in the genetic counseling practice, and compounds the need for large, empirical studies to explicate the clinical utility of whole-genome testing.

Insurance discrimination is an additional area of concern: A study of GWS in newborns discussed some potential participants may have declined participation in the study due to concern for their future healthcare, as at the time of the study the country of study did not have any nondiscrimination laws regarding genetic test results. In total, $35 \%$ of potential participants declined to take part in the study and could create a bias sample population ${ }^{22}$. In order for GWS to be a realistic option for many, legal protections against discrimination for diagnosis or IFs against patients and family members may need to be established before patients will feel confident using GWS.

\section{HRG and Unequal Representation}

The ability of GWS to identify the pathogenicity of a variant is only as good as the panel against which it is measured. In order to establish the risk associated with a variant, there must be a robust reference panel to compare variation within this section of genome in healthy individuals of this population ${ }^{10,88}$. A single Human Reference Genome (HRG) simply will not do. Indeed, a random individual is expected to have a nucleotide variation of 4-5 million when compared to the $\mathrm{HRG}^{62}$. Additionally, when genomes are sequenced using resequencing methods vs de novo assembly, errors are created due to portions of the sequencing genome unable to match with reciprocal portions of the reference genome, resulting in incomplete mapping. One study by Faber-Hammond and Brown found $4.3 \%$ of data from a sample of 45 genomes from individuals from nine distinct population groups were unable to be sequenced against the $\mathrm{HRG}^{89}$. The $H R G$ is not the only reference material to have this problem, however, as many of the studies listed in this paper were conducted on those with European ancestry and only one used a control group of healthy population members. This has created problems in several studies mentioned previously, such as the 2019 Carss et al. study of IRD. Underrepresented populations such as those with African and South Asian ancestry showed a higher rate of VUS due to this lack of representation, and noted a higher rate of genetic diversity as well as consanguinity may have contributed to the difficulty and expose the need for more representation in genetic profiling ${ }^{11}$. Furthermore, populations with high rates of consanguinity present additional diagnostic challenges as the rates of coinheritance of multiple disease and allelic heterogeneity are higher in these populations, already underrepresented in genomic panels, making diagnosis even more difficult ${ }^{90}$.

Only a select number of studies have committed to sampling a diverse population in their cohorts or discussing methods of developing diverse panel of reference genomes. The difficulty of obtaining patients who meet criteria, are willing to participate, and hit a diversity requirement is appreciated. Regardless, it is entirely unacceptable to ignore racial disparity due to the well documented nature of medical racism and GWS must be carefully curated as it is developed to adapt and overcome these racial biases to the absolute best ability.

First attempts at developing diverse reference panels are promising. In an attempt to identify the rate of rare variants in a target population, a study of 1070 genomes at a depth of 30x from healthy Japanese citizens was conducted. The result was a highly sensitive, population specific reference panel of variants. 21 million SNVs and over 3.4 million indels were identified with an estimated false positive rate of $<1 \%{ }^{91}$. This project provides a 
model for the variant panels required for effective future genomic testing.

\section{CONCLUSIONS}

Despite the legitimate hope raised for genome wide sequencing in clinical practice for genetic counselors, significant hang-ups to this process will prevent its use. Despite the current use within some research and experimental clinics, the limitations prove too great for ethical wide spread use. Before the use of GWS can become widespread, there must be large scale studies evaluating the efficacy of GWS in exome analysis as well as provide the information necessary to get GWS testing covered by insurance companies, large-scale population studies creating healthy panels against which variants can be measured, institutional policies relating to consent and incidental findings, and trainings developed for genetic counselors to give effective genomic counseling. While the empirical evidence for the efficacy genome-wide sequencing is critically underdeveloped, it is difficult and possibly unethical to observe the morbidity reduction for vulnerable patient populations and deny them this tool. Still, the risk created by using underdeveloped methodologies which are already predisposed for unequal use and lack regulation is too great to establish use of GWS in clinical practice at this time.

\section{METHODOLOGY}

Initial studies for this literature review were gathered through a methodological search of the PubMed database with the keywords 'genome-wide sequencing' 'genetic counseling' and included studies investigating genome wide sequencing for clinical use and those which provided data or discourse on genetic counseling for genome wide studies. An additional search for "genomic counseling" was conducted, with all articles discussing both genome sequencing and genetic counseling included. Search parameters excluded papers published before the 2014 due to technological limitations and any non-human subjects. Studies were additionally excluded if GWS was conducted using PCR methods, or if GWS comprised less than $10 \%$ of the overall study data. Bibliographies of research papers were scanned for relevant articles and to investigate broad claims. Individual articles were sourced to provide evidence for relevant information not directly related to GWS, EWS, or CMA, these articles were pulled from other bibliographies or articles published $<3$ years ago in high-impact journals from PubMed database searches. Additional searches for specific sections of the paper were conducted in the following sections with the parameters listed below.

Figure 1: PubMed keyword search "diagnostic success rate of exome sequencing" with the filters "human" "english" applied. Studies published before 2011 or not directly conducting research on the diagnostic rate of exome sequencing were excluded.

Figure 2: PubMed keyword search "diagnostic success rate of whole genome sequencing" with the filters "human" "english" applied. Studies published before 2014 or not directly conducting research on the diagnostic rate of genome sequencing were excluded.

\section{REFERENCES}

1. Liu, H.Y.., et al. Diagnostic and clinical utility of whole genome sequencing in a cohort of undiagnosed Chinese families with rare diseases Scientific Reports 9, 19365 (2019)

2. Meynert, A.M., et al. Variant detection sensitivity and biases in whole genome sequencing and exome sequencing Bioinformatics 15, 247 (2014)

3. Bick, D. et al. Case for genome sequencing in infants and children with rare, undiagnosed or genetic diseases $J$ Med Genet 56, 783-791 (2019)

4. Kebschull, J.M. \& Zador, A.M. Sources of PCR-induced distortions in high-throughput sequencing data sets Nucleic Acids Research 21, e143 (2015)

5. Barbitoff, Y.A, et al. Systematic dissection of biases in whole-exome and whole-genome sequencing reveals major determinants of coding sequence coverage Scientific Reports 10, 2057 (2020)

6. Meinberg, J. et al, Clinical sequencing: is WGS better than WES? Hum Gen 135, 359-362 (2016)

7. Meinberg, J. et al New insights into the performance of human whole-exome capture platforms Nucleic Acids Research 43, e76 (2015)

8. Choi, M et al. Genetic Diagnosis by Whole Exome Capture and Massively Parallel DNA Sequencing National Academy of Sciences 106, 19096-19101 (2009)

9. Lelieveld, S.H. et al. Comparison of Exome and Genome Sequencing Technologies for the Complete Capture of Protein-Coding Regions Genomic Medicine 36, 815-822 (2015)

10. Sanghvi, R.V. et al. Characterizing reduced coverage regions through comparison of exome and genome sequencing data across 10 centers Clinical Genetics 20, 855-866 (2018)

11. Carss K.J. et al. Comprehensive Rare Variant Analysis via Whole-Genome Sequencing to Determine the Molecular Pathology of Inherited Retinal Disease J Med Genet 100, 75-90 (2017)

12. Lupski, J.R. Structural Variation Mutagenesis of the Human Genome: Impact on Disease and Evolution Environ Mol Mutagen 56, 419-436 (2015)

13. Rehm, H.L. et al. ACMG clinical laboratory standards for next-generation sequencing Genetics in Medicine 15, 733-747 (2013)

14. Xue Y. et al. Solving the molecular diagnostic testing conundrum for Mendelian disorders in the era of next-generation sequencing: single-gene, gene panel, or exome/genome sequencing Genetics in Medicine 17, 444-451 (2015)

15. Marshall C.R. et al. Best practices for the analytical validation of clinical whole-genome sequencing intended for the diagnosis of germline disease Genomic Medicine 5, (2020)

16. Lionel A.C. et al. Improved diagnostic yield compared with targeted gene sequencing panels suggests a role for 
whole-genome sequencing as a first-tier genetic test Genetics in Medicine 20, 435-343 (2018)

17. Clark M.M. et al. Meta-analysis of the diagnostic and clinical utility of genome and exome sequencing and chromosomal microarray in children with suspected genetic diseases Genomic Medicine 3, (2018)

18. Gross A.M. et al. Copy-number variants in clinical genome sequencing: deployment and interpretation for rare and undiagnosed disease Genetics in Medicine 21, 1121-1131 (2019)

19. Belkadi A. et al. Whole-genome sequencing is more powerful than whole-exome sequencing for detecting exome variants Proceedings of the National Academy of Sciences of the United States of America 112, 5473-5478 (2015)

20. Ellingford J.M. et al.Whole Genome Sequencing Increases Molecular Diagnostic Yield Compared with Current Diagnostic Testing for Inherited Retinal Disease Ophthalmology 123, 1143-1150 (2016)

21. Liao C. et al. Prenatal diagnosis of congenital heart defect by genome-wide high-resolution SNP array Prenatal Diagnosis 34, 858-863 (2014)

22. Stavropoulos D.J. et al. Whole-genome sequencing expands diagnostic utility and improves clinical management in paediatric medicine Genomic Medicine 1, 15012 (2016)

23. Wray N.R. et al. Genome-wide association analyses identify 44 risk variants and refine the genetic architecture of major depression Nature Genetics 50, 668-681 (2018)

24. Ferreira M.A. et al. Genome-wide association and transcriptome studies identify target genes and risk loci for breast cancer Nature Communications 10, 1741 (2019)

25. Alfares A. et al. Whole-genome sequencing offers additional but limited clinical utility compared with reanalysis of whole-exome sequencing Genetics in Medicine 20, 1328-1333 (2018)

26. Wilson B.J., Miller F.A., Rousseau F. Controversy and debate on clinical genomics sequencing - paper 1: genomics is not exceptional: rigorous evaluations are necessary for clinical applications of genomic sequencing Journal of Clinical Epidemiology 29, 4-6 (2017)

27. Rodenburg R.J. The functional genomics laboratory: functional validation of genetic variants Journal of Inherited Metabolic Disease 41, 297-307 (2018)

28. Zhang N. et al. Performance characterization of PCR-free whole genome sequencing for clinical diagnosis (2020)

29. Yuen R. et al. Whole-genome sequencing of quartet families with autism spectrum disorder Nature Medicine 21, 185-195 (2014)

30. Chakravorty S., Hegde M. Inferring the effect of genomic variation in the new era of genomics Human Mutation 39, (2018)

31. Taylor J.C. et al. Factors influencing success of clinical genome sequencing across a broad spectrum of disorders Nature Genetics 47, 717-730 (2015)

32. Willing L.K. et al. Whole-genome sequencing for identification of Mendelian disorders in critically ill infants: a retrospective analysis of diagnostic and clinical findings Lancet Respir Med. 3, 377-387 (2015)

33. Costain G. et al. Periodic reanalysis of whole-genome sequencing data enhances the diagnostic advantage over standard clinical genetic testing European Journal of Human Genetics 26, 740-744 (2018)

34. Hiatt S.M. Systematic reanalysis of genomic data improves quality of variant interpretation Clinical Genetics 94, 174-178 (2018)

35. Liu P. et al. Reanalysis of Clinical Exome Sequencing Data New England Journal of Medicine 380, 2478-2480(2019)

36. Salfati E.L. et al. Re-analysis of whole-exome sequencing data uncovers novel diagnostic variants and improves molecular diagnostic yields for sudden death and idiopathic diseases Genome Medicine 11, (2019)

37. Soutza N. The ENCODE project Nature Methods 9, 1046 (2012)

38. Yang J., Aldi M. Mapping and Making Sense of Noncoding Mutations in the Genome Cancer Res 79, 4309-4314 (2019)

39. Scacheri C.A, Scacheri P.C. Mutations in the non-coding genome Curr Opin Pediatr. 27, 659-664 (2015)

40. Nagasaki M. et al. Rare variant discovery by deep whole-genome sequencing of 1,070 Japanese individuals Nature Communications 6, 8018 (2015)

41. Horn S. et al. TERT Promoter Mutations in Familial and Sporadic Melanoma American Association for the Advancement of Science 339, 959-961 (2013)

42. Iglesias A. et al. The usefulness of whole-exome sequencing in routine clinical practice Genetics in Medicine 16, 922-932 (2014)

43. Saunders C.J. et al. Rapid Whole-Genome Sequencing for Genetic Disease Diagnosis in Neonatal Intensive Care Units Sci Transl Med. 4, (2012)

44. Socchia A. et al. Clinical whole genome sequencing as a first-tier test at a resource-limited dysmorphology clinic in Mexico Genomic Medicine 5, (2019)

45. Farnaes L.et al. Rapid whole-genome sequencing decreases infant morbidity and cost of hospitalization, Genomic Medicine 10, (2018)

46. Willig L.K. et al. Whole-genome sequencing for identification of Mendelian disorders in critically ill infants: a retrospective analysis of diagnostic and clinical findings Lancet Respir Med. 3, 377-387 (2015)

47. Petrikin J.E. et al. Rapid whole genome sequencing and precision neonatology Seminars in Perinatology 5, 623-631 (2015)

48. Cohn I. et al. Genome sequencing as a platform for pharmacogenetic genotyping: a pediatric cohort study Genomic Medicine 2, (2017)

49. Hayashi S. et al. ALPHLARD: a Bayesian method for analyzing HLA genes from whole genome sequence data. BMC Genomics 19, (2018)

50. Friedman J. et al. Genomic newborn screening: public health policy considerations and recommendations $B M C$ Medical Genomics 10, (2017) 
51. Sabbagh R., Veyver I.B. The current and future impact of genome-wide sequencing on fetal precision medicine Human Genetics 139, 1121-1130 (2020)

52. Veyver I.B., Eng C.M. Genome-Wide Sequencing for Prenatal Detection of Fetal Single-Gene Disorders Cold Spring Harb Prospect Med 5 (2015)

53. Vermessche J.R., Voet T., Devriendt K., Prenatal and pre-implantation genetic diagnosis Genetics 17, 634-656 (2016)

54. Liu H.Y. et al. Diagnostic and clinical utility of whole genome sequencing in a cohort of undiagnosed Chinese families with rare diseases Scientific Reports 9, (2019)

55. Matias M. et al. Comparison of medical management and genetic counseling options pre- ${ }^{-}$and post ${ }^{-}$whole exome sequencing for patients with positive and negative results $J$. Genet Couns 28, 182-193 (2019)

56. Patch C., Middleton A. Genetic counselling in the era of genomic medicine British Medical Bulletin 126, 27-36 (2018)

57. Campion, M. et al. Genomic education for the next generation of health-care providers GENETICS in MEDICINE 21, 2422-2430 (2019)

58. Savard J. et al. From Expectations to Experiences: Consumer Autonomy and Choice in Personal Genomic Testing AJOB Empirical Bioethics 11, 63-76 (2020)

59. Smith E.E., et al. Genetic counseling considerations with rapid genome-wide sequencing in a neonatal intensive care unit J Genet Couns 28, 263-272 (2019)

60. Ayres S. et al. Genetic counseling in pediatric acute care: Reflections on ultra-rapid genomic diagnoses in neonates $J$ Genet Couns 28, 273-282 (2019)

61. Mcnamara E.F. et al. Cases from the Undiagnosed Diseases Network: The continued value of counseling skills in a new genomic era J Genet Couns 28, 194-201 (2019)

62. Elliott A.M., Friedman J.M. The importance of genetic counselling in genome-wide sequencing Genetics 19, 735-739 (2018)

63. Marshall, C.R, et al. Best practices for the analytical validation of clinical whole-genome sequencing intended for the diagnosis of germline disease Genomic Medicine 47, (2020)

64. Birch, P. et al. DECIDE: a Decision Support Tool to Facilitate Parents' Choices Regarding Genome-Wide Sequencing J Genet Counsel 25, 1298-1308 (2016)

65. Richards S. et al. Standards and guidelines for the interpretation of sequence variants: a joint consensus recommendation of the American College of Medical Genetics and Genomics and the Association for Molecular Pathology GENETICS in MEDICINE 17, 405-424 (2015)

66. Holm I.A. et al. The BabySeq project: implementing genomic sequencing in newborns BMC Pediatrics 18, (2018)

67. Metcalfe S.A. Genetic counselling, patient education, and informed decision-making in the genomic era Seminars in Fetal \& Neonatal Medicine 23, 142-149 (2018)
68. Starkweather, A.R. et al. Strengthen federal regulation of laboratory developed and direct-to-consumer genetic testing Nurs Outlook 66, 1010-104 (2018)

69. Schmidt J.L., Mass R., Altmeyer S.R. Genetic counseling for consumer-driven whole exome and whole genome sequencing: A commentary on early experiences $J$ Genet Couns 28, 449-455 (2019)

70. Boycott K. et al. The clinical application of genome-wide sequencing for monogenic diseases in Canada: Position Statement of the Canadian College of Medical Geneticists $J$ Med Genet 0, 1-7 (2015)

71. Machini K., et al. Genetic Counselors' Views and Experiences with the Clinical Integration of Genome Sequencing J Genet Counsel 23, 496-505 (2014)

72. Samuel G.N., Healthcare professionals' and patients' perspectives on consent to clinical genetic testing: moving towards a more relational approach BMC Medical Ethics 18, (2017)

73. Hitchcock E.C., Elliott A.M., Shortened consent forms for genome-wide sequencing: Parent and provider perspectives Med Genet Genomic Med 8, (2020)

74. Sanderson S.C., et al. Opening the "black box" of informed consent appointments for genome sequencing: a multisite observational study GENETICS in MEDICINE 21, 1083-1091 (2019)

75. Vinatzer B.A., et al. Cyberbiosecurity Challenges of PAthogen Genome Databases Frontiers in Bioengineering and Biotechnology 7, (2019)

76. Green R.C., et al. ACMG Recommendations for Reporting of Incidental Findings in Clinical Exome and Genome Sequencing Genet Med. 15, 565-574 (2013)

77. Li K.C., et al. Parents' Perspectives on Supporting Their Decision Making in Genome-Wide Sequencing Journal of Nursing Scholarship 48, 265-275 (2016)

78. Manrique de Lara A., et al. Ethical issues in susceptibility genetic testing for late-onset neurodegenerative diseases $A M$ J Med Genet 1, 1-13

79. Arora S. et al. Clinical and Counseling Experiences of Early Adopters of Whole Exome Sequencing J Genet Counsel 25, 337-343 (2016)

80. Smith L.A., et al. Reporting Incidental Findings in Clinical Whole Exome Sequencing: Incorporation of the 2013 ACMG Recommendations into Current Practices of Genetic Counseling J Genet Counsel 24, 654-662 (2015)

81. Lohn Z., Adam S., Birch P.H., Friedman J.M. Incidental Findings from Clinical Genome-Wide Sequencing: A Review J Genet Counsel 23, 463-473 (2014)

82. Grones, G. Top 10 health insurance companies in the US Insurance Business (2021)

83. UnitedHealthcare Community Plan. Whole Exome and Whole Genome Sequencing Medical Policy (2021)

84. Anthem BlueCross. Whole Genome Sequencing, Whole Exome Sequencing, Gene Panels, and Molecular Profiling Medical Policy (2020)

85. Aetna. Genetic Testing Medical Clinical Policy Bulletins (2020) 
86. Cigna. Whole Exome and Whole Genome Sequencing Medical Coverage Policy (2021)

87. Humana. Genetic Testing Medical Coverage Policy (2020)

88. Fresard L., Montgomery S.B. Diagnosing rare diseases after the exome Cold Spring Harb Mol Case Stud 4, (2018)

89. Faber-Hammond J.J., Brown K.H. Anchored pseudo-de novo assembly of human genomes identifies extensive sequence variation from unmapped sequence reads $\mathrm{Hum}$ Genet 135, 727-740 (2016)

90. Shalev S.A. Characteristics of genetic diseases in consanguineous populations in the genomic era: Lessons from Arab communities in North Israel Clinical Genetics 95, 3-9 (2019)

91. Nagasaki M., et al. Rare variant discovery by deep whole-genome sequencing of 1,070 Japanese individuals Nature Communications 6, (2015) 